Bemerkungen. Die Beobachtungen sind bei 200-maliger Vergrößerung angestellt. - Sept. I x. Der Komet erscheint als runde Nebelmasse mit Andeutung einer Verdichtung. - Sept. I 2. Aussehen des Kometen wie am Tage vorher. - Sept. 13. Aussehen des Kometen unverändert. - Sept. I 5. Kein Vergleichstern. - Sept. 18. Komet heller mit merklicher Verdichtung. Bei Beginn der Beobachtung bewölkte es sich. - Sept. 2 I und 22 . Aussehen wie Sept. I 8.

Pulkowa, I 922 Mai.

F. Renz.

\title{
Beobachtungen des Kometen 1921 a (Reid)
}

\begin{tabular}{|c|c|c|c|c|c|c|c|c|c|c|c|c|c|c|c|c|c|c|c|}
\hline \multicolumn{2}{|c|}{1921} & \multicolumn{3}{|c|}{ M. Z. Stockbolm } & \multirow{2}{*}{\multicolumn{2}{|c|}{$\begin{array}{c}\Delta \alpha \\
+\mathrm{I}^{\mathrm{m}} 50.10\end{array}$}} & \multirow{2}{*}{$-3^{\prime}$} & \multirow{2}{*}{$\begin{array}{l}\Delta \delta \\
32 \% 0\end{array}$} & \multirow{2}{*}{$\begin{array}{l}\text { Vgl. } \\
6,5\end{array}$} & \multirow{2}{*}{\multicolumn{3}{|c|}{$\begin{array}{c}\alpha \text { app. } \\
20^{\mathrm{h}} 29^{\mathrm{m}} 37^{\mathrm{s}} \cdot 91\end{array}$}} & \multirow{2}{*}{$\begin{array}{l}\log p \cdot \Delta \\
9 \cdot 4^{2} 5 n\end{array}$} & \multicolumn{3}{|c|}{ Oapp. } & \multirow{2}{*}{$\begin{array}{l}\log p \cdot 1 \\
0.867\end{array}$} & \multicolumn{2}{|c|}{ Red. ad 1. app. } \\
\hline Apri & 9 & I $5^{h}$ & & $24: 5$ & & & & & & & & & & +4 & $5^{\prime}$ & 22.9 & & +0.94 & +2.3 \\
\hline & I 5 & I 4 & 22 & I 2.7 & +2 & 4.55 & +5 & 36.9 & 7,10 & 20 & 34 & 7.06 & $9.463 \mathrm{n}$ & +15 & I I & 14.0 & 0.836 & $+\mathrm{r} .05$ & -0.5 \\
\hline & I 6 & I 4 & $3 \mathrm{I}$ & 33.2 & -0 & 32.40 & -7 & 0.6 & I 1,5 & 20 & 35 & 0.97 & $9.459 n_{0}$ & +17 & 28 & I 8.9 & 0.823 & +1.06 & -1.0 \\
\hline & 25 & I 2 & 36 & 27.0 & +0 & $3^{8.33}$ & -2 & 6.8 & 10,6 & 20 & 47 & 21.72 & 9.6 I $8 n$ & +43 & 43 & $5 \mathrm{I} . \mathrm{I}$ & 0.733 & + I. I 7 & -6.1 \\
\hline & 26 & I 2 & $4^{6}$ & 22.2 & -2 & 57.82 & +5 & 11.5 & 8,5 & 20 & 49 & $4 I \cdot 55$ & $9.645 n$ & +47 & I 5 & 58.4 & 0.69 .3 & +1.17 & -6.4 \\
\hline & 5 & I 3 & 10 & 47.9 & +5 & 33.89 & +6 & 3.8 & 7,5 & $2 \mathrm{I}$ & $5^{8}$ & I 4.33 & $0.16 \mathrm{In}$ & +78 & 3 & 44.6 & $0.16 \mathrm{I}$ & +0.92 & -7.6 \\
\hline & 13 & 12 & $3 I$ & 14.0 & +0 & 26.07 & $-I$ & 27.5 & 10,5 & 6 & 47 & 27.23 & 0.065 & +79 & $3^{8}$ & I 8.9 & 0.667 & $-0.6 \mathrm{I}$ & +3.0 \\
\hline & 14 & I 2 & 23 & 54.6 & +0 & $55.4 \mathrm{I}$ & +3 & 6.1 & 8,6 & 7 & 3 & 47.69 & 0.024 & +77 & $5^{\circ}$ & 48.3 & 0.657 & -0.27 & +2.9 \\
\hline
\end{tabular}

Mittlere Örter der Vergleichsterne.

\begin{tabular}{|c|c|c|c|c|c|c|}
\hline \multirow{2}{*}{\multicolumn{3}{|c|}{$\begin{array}{c}\alpha_{1921.0} \\
20^{\mathrm{h}} 2_{2} \mathrm{~m}_{4} 6^{\mathrm{s}} 87\end{array}$}} & \multicolumn{3}{|c|}{81921.0} & Autorität \\
\hline & & & $+4^{\circ}$ & & 52.6 & Alb 7 I 52 \\
\hline 20 & $3^{2}$ & I. 46 & $+\mathrm{r}_{5}$ & 5 & 37.6 & Berl A 827 I \\
\hline 20 & 35 & $3^{2} \cdot 31$ & +17 & 35 & 20.5 & $\begin{array}{r}8309 \\
\end{array}$ \\
\hline 20 & 46 & 42.22 & +43 & 46 & 4.0 & Bo I 4724 \\
\hline 20 & 52 & 38.20 & +47 & IO & $53 \cdot 3$ & $" \mathrm{I} 4855$ \\
\hline 2 I & $5^{2}$ & $39 \cdot 5^{2}$ & +77 & 57 & 48.4 & $\mathrm{Gr} p h+77^{\circ} 7598$ \\
\hline 6 & 47 & 1.77 & +79 & 39 & $43 \cdot 4$ & v. m. Grph $+79^{\circ} 2414$ \\
\hline 7 & 2 & $5^{2.55}$ & +77 & 47 & $39 \cdot 3$ & $\mathrm{Grph}+77^{\circ} 2913$ \\
\hline
\end{tabular}

herum schwächerer Nebel, kein Schweif. - April 2 5. Kern $10^{\mathrm{m}}$, ungewiß ob granuliert, darum Verdichtung und weiter außen

schwächere Koma. - April 26. Beob. recht befriedigend. Mai I 3. Mondschein und Nordlicht, wodurch Beob. recht schwierig. - Mai 14. Komet immer noch so hell wie im Perihel, deutlicher Kern, Fadenbeleuchtung erscheint schwach infolge des hellen Himmelsgrundes.

Photographische Aufnahmen. Mai I. Feiner, fast gradliniger Schweif, $3^{\circ}$ beinahe im Parallel, kurzer $\left(0^{\circ} 2\right)$ Nebenschweif, im Gegensatz zum Hauptschweif an der Basis breit, und spitzenförmig verlaufend, erscheint mehr als ein Ausläufer von der äußeren Koma, fast entgegengesetzt der scheinbaren Bewegung des Kometen, Winkel der beiden Schweife scheinbar ungefähr $50^{\circ}$, Platte 1708 .

Stockholm, i 922 April I 5.

K. Bohlin.

\section{Beobachtungen des Kometen 1922 b (Skjellerup).}

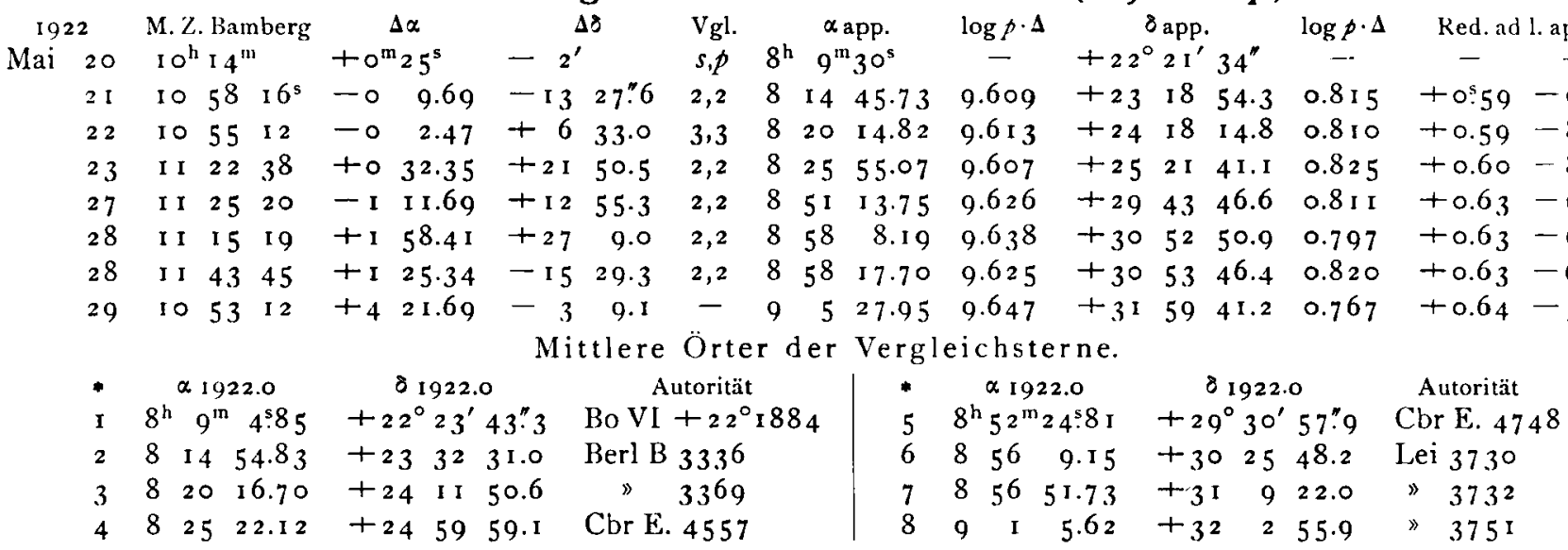

Die Beobachtung vom 29. Mai ist mit dem Lamellenmikrometer (Beob. Dr. E. Heise) erhalten, die übrigen von mir mit dem Heliometer. Die Messungen am Heliometer waren äußerst schwierig bei der Schwäche der 6' großen Kometenscheibe. Ier Ort am 20. Mai ist eine Einschätzung in die Sternkarte.

Bamberg, Remeis-Sternwarte, 1922 Juni 2.

E. Hartwig.

Zwei neue, nahe aneinander gelegene, veränderliche Sterne vom Clustertypus. Von $R$. Furuhjelm.

Bei der Vergleichung einiger Aufnahmen unserer Himmelskartenregion Nr. $698\left(\alpha=16^{\mathrm{h}} 35^{\mathrm{m}}, \delta=+41^{\circ} \circ^{\prime}\right)$ fand ich $z$ wei nahe aneinander liegende veränderliche Sterne. Um die Veränderlichkeit näher zu untersuchen, machte ich nachher eine Reihe von Aufnahmen, und es erwies sich, daß beide Sterne kurzperiodische Veränderliche vom Clustertypus sind. Die Koordinaten der Sterne wurden durch Anschluß an die auf den Platten vorkommenden AG-Sterne bestimmt, und 